\title{
PROBABLISTIC CONVERGENCE SPACES AND REGULARITY
}

\author{
P. BROCK and D.C. KENT \\ Department of Pure and Applied Mathematics \\ Washington State University \\ Pullman, WA 99164-3113, U.S.A.
}

(Received February 5, 1996 and in revised form May 20, 1996)

\begin{abstract}
The usual definition of regularity for convergence spaces can be characterized by a diagonal axiom $\mathbf{R}$ due to Cook and Fischer. The generalization of $\mathbf{R}$ to the realm of probabilistic convergence spaces depends on a $t$-norm $\mathrm{T}$, and the resulting axiom $\mathbf{R}_{T}$ defines "T-regularity", which is the primary focus of this paper. We give several characterizations of $T$-regularity, both in general and for specific choices of $T$, and investigate some of its basic properties.

KEY WORDS AND PHRASES. convergence space, probabilistic convergence space, $t$-norm, T-regular space, $\mathrm{T}$-approachable space, category

1991 AMS SUBJECT CLASSIFICATION CODES: 54 A 05, 54 A 20, 54 D 10, 54 E 70
\end{abstract}

\section{INTRODUCTION.}

Probabilistic convergence spaces were introduced in 1989 by L. Florescu [4] in terms of nets, and were later reformulated using filters in [8]. Probabilistic convergence spaces are a natural extension of probabilistic metric spaces [9]; the fundamental idea is to assign a numerical probability to the convergence of a given filter to a given point. From a categorical perspective, the category PCS of probabilistic convergence spaces is cartesian closed and hereditary (i.e., a quasitopos).

In [3], Cook and Fischer investigated two diagonal conditions for convergence spaces, we call them $\mathbf{F}$ and $\mathbf{R}$, which are in a natural way dual to each other. A convergence space is topological iff it satisfies $\mathbf{F}$, and regular iff it satisfies $\mathbf{R}$. In [8], $\mathbf{F}$ was generalized relative to an arbitrary $t$-norm $T$ to an axiom $\mathbf{F}_{T}$ for probabilistic convergence spaces, and it was shown in [2] that the full subcategory of PCS determined by the left-continuous probabilistic limit spaces satisfying $\mathbf{F}_{T}$ for a strict $t$-norm $\mathrm{T}$ is isomorphic to R. Lowen's category AP of approach spaces [7]. Thus we define probabilistic convergence spaces satisfying $\mathrm{F}_{T}$ to be $T$-approachable.

Our goal in this paper is to study the dual axiom $\mathbf{R}_{T}$ in the category PCS. Probabilistic convergence spaces satisfying $\mathbf{R}_{T}$ are defined to be $T$-regular. If $\mathrm{T}$ and $\mathrm{T}^{\prime}$ are $t$-norms such that $\mathrm{T} \leq \mathrm{T}^{\prime}$, then $\mathrm{T}^{\prime}$-regularity implies T-regularity. Indeed, the largest $t$-norm, denoted by $\check{T}$, induces the strongest type of "T-regularity," which is equivalent to "componentwise regularity". Likewise the smallest $t$-norm, $\hat{T}$, induces what we naturally call "weak regularity".

For an arbitrary $t$-norm $T$, we give several "traditional" characterizations of $T$-regularity in PCS and some of its subcategories, along with examples to show how $T$-regularity depends on the choice of $T$. We also show that $T$-regularity is preserved under the formation of initial structures, thereby demonstrating that the category $\mathrm{R}_{T} \mathrm{PCS}$ of $T$-regular probabilistic convergence spaces is 
bireflectively embedded in PCS. It should also be noted that the category RCONV of regular convergence spaces is bicoreflectively embedded in $\mathrm{R}_{T}$ PCS for an arbitrary $t$-norm $T$.

In the last section, we show that in certain subcategories of PCS, the axiom $\mathbf{F}_{T}$ and $\mathbf{R}_{T}$ can be stated entirely in terms of ultrafilters, which in some situations results in an appreciable simplification.

\section{CONVERGENCE SPACES.}

Let $X$ be a set, $\mathbf{F}(X)$ the set of all filters on $X, \mathrm{U}(X)$ the set of all ultrafilters on $X$, and $2^{X}$ the power set of $X$. For each $x \in X, \dot{x}$ denotes the fixed ultrafilter generated by $x$.

Definition 1.1. A function $q: \mathbf{F}(X) \rightarrow 2^{X}$ is called a convergence structure on $X$ if it satisfies the following axioms.

$\left(\mathrm{C}_{1}\right) x \in q(\dot{x})$, for all $x \in X$;

$\left(\mathrm{C}_{2}\right) \mathcal{F} \subseteq \mathcal{G} \Rightarrow q(\mathcal{F}) \subseteq q(\mathcal{G})$

$\left(\mathrm{C}_{3}\right) x \in q(\mathcal{F}) \Rightarrow x \in q(\mathcal{F} \cap \dot{x})$.

If $q$ is a convergence structure on $X$, the pair $(X, q)$ is called a convergence space, and " $x \in q(\mathcal{F})$ " will usually be written $" \mathcal{F} \stackrel{q}{\rightarrow} x$ " (F $q$-converges to $x)$. A function $f:(X, q) \rightarrow(Y, p)$ between convergence spaces is continuous if $f(\mathcal{F}) \stackrel{p}{\rightarrow} f(x)$ whenever $\mathcal{F} \stackrel{q}{\rightarrow} x$. If $p$ and $q$ are convergence structures on $X$ and $f:(X, q) \rightarrow(X, p)$ is continuous, where $f$ is the identity map on $X$, then we write $p \leq q$ ( $p$ is coarser than $q$, or $q$ is finer than $p$ ).

For a convergence space $(X, q)$, consider the following additional axioms.

$\left(\mathrm{C}_{\mathbf{4}}\right) \mathcal{F} \stackrel{\mathfrak{q}}{\rightarrow} x$ and $\mathcal{G} \stackrel{q}{\rightarrow} x \Rightarrow \mathcal{F} \cap \mathcal{G} \stackrel{q}{\rightarrow} x$.

$\left(\mathrm{C}_{5}\right)$ If $\mathcal{G} \in \mathbf{F}(X)$ and $\mathcal{F} \stackrel{\mathfrak{q}}{\rightarrow} x$, for all $\mathcal{F} \in \mathrm{U}(X)$ such that $\mathcal{G} \subseteq \mathcal{F}$, then $\mathcal{G} \stackrel{q}{\rightarrow} x$.

$\left(\mathrm{C}_{6}\right) \mathcal{V}_{q}(x) \stackrel{q}{\rightarrow} x$, for all $x \in X$, where $\mathcal{V}_{q}(x)$ is the $q$-neighborhood filter at $x$, defined by

$\mathcal{V}_{q}(x)=\bigcap\{\mathcal{F} \in \mathbf{F}(X): \mathcal{F} \stackrel{q}{\rightarrow} x\}$.

A convergence structure $q$ on $X$ satisfying $\left(\mathrm{C}_{4}\right)$ (respectively, $\left(\mathrm{C}_{5}\right),\left(\mathrm{C}_{6}\right)$ ) is called a limit structure (respectively, pseudotopology, pretopology) and $(X, q)$ is called a limit space (respectively, pseudotopological space, pretopological space).

With every convergence space $(X, q)$, there is an associated closure operator $c l_{q}: 2^{X} \rightarrow 2^{X}$ defined by $\operatorname{cl}_{q} A=\{x \in X: \exists \mathcal{F} \stackrel{q}{\rightarrow} x$ such that $A \in \mathcal{F}\}$, for all $A \subseteq X .(X, q)$ is said to be regular if $\mathcal{F} \stackrel{q}{\rightarrow} x$ implies $c l_{q} \mathcal{F} \stackrel{q}{\rightarrow} x$, where $c l_{q} \mathcal{F}$ is the filter generated by $\left\{c l_{q} F: F \in \mathcal{F}\right\}$. A convergence space is said to be topological if $q$-convergence coincides with that relative to some topology on $X$; in this case it is customary to identify that topology with $q$.

It is an interesting (and apparently not well-known) fact that the convergence properties "regular" and "topological" are in a very natural sense dual to each other, since they can be characterized by means of dual axioms, which we call $\mathbf{F}$ and $\mathbf{R}$, due to C.H. Cook and H.R. Fischer [3]. Let $X$ and $J$ be non-empty sets, $\mathcal{F} \in \mathbf{F}(J)$, and $\sigma: J \rightarrow \mathbf{F}(X)$. We define

$$
\kappa \sigma \mathcal{F}=\bigcup_{F \in \mathcal{F}} \bigcap_{y \in F} \sigma(y)
$$

$\kappa$ is called the "compression operator for $\mathcal{F}$ relative to $\sigma$." Note that if $\mathcal{F} \in \mathbf{U}(J)$, and $\sigma(y) \in \mathbf{U}(X)$ for all $y \in J$, then $\kappa \sigma \mathcal{F} \in \mathbf{U}(X)$. We can now define the axioms $\mathbf{F}$ and $\mathbf{R}$.

F: Let $J$ be a non-empty set, $\psi: J \rightarrow X$, and let $\sigma: J \rightarrow \mathbf{F}(X)$ have the property that $\sigma(y) \stackrel{q}{\rightarrow} \psi(y)$, for all $y \in J$. If $\mathcal{F} \in \mathbf{F}(J)$ is such that $\psi(\mathcal{F}) \stackrel{q}{\rightarrow} x$, then $\kappa \sigma \mathcal{F} \stackrel{q}{\rightarrow} x$. 
$\mathbf{R}$ : Let $J$ be a non-empty set, $\psi: J \rightarrow X$, and let $\sigma: J \rightarrow \mathbf{F}(X)$ have the property that $\sigma(y) \stackrel{q}{\rightarrow} \psi(y)$, for all $y \in J$. If $\mathcal{F} \in \mathbf{F}(J)$ is such that $\kappa \sigma \mathcal{F} \stackrel{q}{\rightarrow} x$, then $\psi(\mathcal{F}) \stackrel{q}{\rightarrow} x$.

The next proposition summarizes previously mentioned results pertaining to these axioms. The first assertion is proved in [8], the second in [1] and [3].

Proposition 1.2. Let $(X, q)$ be a convergence space.

(1) $(X, q)$ is topological if and only if it satisfies $\mathbf{F}$

(2) $(X, q)$ is regular if and only if it satisfies $\mathbf{R}$

Let $\mathbf{F}^{*}$ and $\mathbf{R}^{*}$ denote the axioms obtained when " $\mathbf{F}(X)$ " is replaced by " $U(X)$ " in $\mathbf{F}$ and $\mathbf{R}$, respectively. Obviously, $\mathbf{F} \Rightarrow \mathbf{F}^{*}$ and $\mathbf{R} \Rightarrow \mathbf{R}^{*}$. The next proposition is proved in [5].

Proposition 1.3. For convergence spaces, $\mathbf{F} \Longleftrightarrow \mathbf{F}^{*}$ and $\mathbf{R} \Longleftrightarrow \mathbf{R}^{*}$.

Let CONV denote the category of convergence spaces and continuous maps. Let RCONV be the full subcategory of CONV determined by the regular objects and TOP the full subcategory of CONV determined by the topological objects. It is well known that both RCONV and TOP are bireflective subcategories of CONV, since the properties "regular" and "topological" are both preserved under formation of initial structures.

\section{PROBABILISTIC CONVERGENCE SPACES.}

This section is mainly a review of relevant definitions and theorems from [2] and [8]. Let $I$ denote the closed unit interval $[0,1]$.

Definition 2.1. A probabilistic convergence structure $\mathbf{q}$ on $X$ is a function $\mathbf{q}: \mathbf{F}(X) \times I \rightarrow 2^{X}$ which satisfies:

$\left(\mathrm{PCS}_{1}\right)$ For each $\mu \in I, q(\mathcal{F}, \mu)=q_{\mu}(\mathcal{F})$, where each $q_{\mu}$ is a convergence structure on $X$;

$\left(\mathrm{PCS}_{2}\right)$ When $\mu=0, q_{\mu}$ is the indiscrete topology;

$\left(\mathrm{PCS}_{3}\right)$ If $\mu \leq \nu$, then $q_{\mu} \leq q_{\nu}$.

If $\mathbf{q}$ is a probabilistic convergence structure on $X$, the pair $(X, \mathbf{q})$ is called a probabilistic convergence space. We will usually write $q=\left(q_{\mu}\right)$, where it is understood that $\mu$ ranges through $I$; the $q_{\mu}$ 's are called the "component convergence structures." If $q=\left(q_{\mu}\right)$ where each $q_{\mu}$ is a limit structure (respectively, pseudotopology, pretopology, topology), then $(X, \mathbf{q})$ is called a probabilistic limit space (respectively, probabilistic pseudotopological space, probabilistic pretopological space, probabilistic topological space).

If $(X, \mathbf{q})$ is a probabilistic convergence space, $\mathcal{F} \in \mathbf{F}(X)$, and $x \in X$, then $\mu=\sup \left\{\nu \in I: \mathcal{F} \stackrel{q_{\nu}}{\longrightarrow}\right.$ $x\}$ is interpreted as the probability that $\mathcal{F}$ q-converges to $x$.

A probabilistic convergence space $(X, q)$ is left-continuous if, for each $\mu \in(0,1], q_{\mu}=\sup \left\{q_{\nu}\right.$ : $\nu<\mu\}$, and constant if, for each $\mu \in(0,1], q_{\mu}=q_{1}$.

If $(X, \mathbf{q}),(Y, \mathbf{p})$ are probabilistic convergence spaces and $f: X \rightarrow Y$ is a map, then $f:(X, \mathbf{q}) \rightarrow$ 
$(Y, \mathrm{p})$ is said to be contınuous if $f:\left(X, q_{\mu}\right) \rightarrow\left(Y, p_{\mu}\right)$ is continuous, for each $\mu \in I$. We denote by PCS the category whose objects are probabilistic convergence spaces and whose morphisms are continuous maps. Some full subcategories of PCS which are of interest are the following:

PPSS (objects are probabilistic pseudotopological spaces)

PPRS (objects are probabilistic pretopological spaces)

PTS (objects are probabilistic topological spaces)

Furthermore, the full subcategory of PCS determined by the constant objects is isomorphic to CONV. We note that CONV, PTS, PPRS, and PPSS are bireflectively embedded in PCS; CONV is also bicoreflectively embedded in PCS.

The notion of " $t$-norm," which is vital in the study of probabilistic metric spaces (see [9]), also plays an important role in the study of probabilistic convergence spaces. We shall summarize some facts about $t$-norms which are relevant to this paper; for further information the reader is referred to [2] or [9].

A $t$-norm is a binary operator $T: I^{2} \rightarrow I$ which is associative, commutative, increasing in each variable, and satisfies $T(\mu, 1)=\mu$, for all $\mu \in I$. Let $\mathrm{T}$ be the set of all $t$-norms, with pointwise partial order. $\mathbf{T}$ contains a largest member $\check{T}$, defined by $\check{T}(\mu, \nu)=\min \{\mu, \nu\}$, and a smallest member $\hat{T}$, defined by

$$
\hat{T}(\mu, \nu)= \begin{cases}\mu, & \text { if } \nu=1 \\ \nu, & \text { if } \mu=1 \\ 0, & \text { otherwise. }\end{cases}
$$

A $t$-norm $T \in \mathbf{T}$ is said to be strict if there is a surjective, strictly decreasing map $S: I \rightarrow[0, \infty]$ such that $T(\mu, \nu)=S^{-1}(S(\mu)+S(\nu))$. Neither $\check{T}$ nor $\hat{T}$ is strict; an example of a strict $t$-norm is $T(\mu, \nu)=\mu \nu$, where $S(\mu)=-\log \mu$.

Let $(X, \mathbf{q})$ be a probabilistic convergence space, and let $T \in \mathbf{T}$. We define two axioms for $(X, \mathbf{q})$ relative to $T$ which are derived in an obvious way from the axioms $\mathbf{F}$ and $\mathbf{R}$ of Section 1 .

$\mathbf{F}_{T}:$ Let $\mu, \nu \in I$. Let $J$ be any non-empty set, $\psi: J \rightarrow X$ and $\sigma: J \rightarrow \mathbf{F}(X)$ be such that $\sigma(y) \stackrel{q_{\nu}}{\rightarrow} \psi(y)$, for each $y \in J$. If $\mathcal{F} \in \mathbf{F}(J)$ and $\psi \mathcal{F} \stackrel{q_{\mu}}{\rightarrow} x$, then $\kappa \sigma \mathcal{F} \stackrel{q_{T(\mu, \nu)}}{\longrightarrow} x$.

$\mathbf{R}_{T}$ : Let $\mu, \nu \in I$. Let $J$ be any non-empty set, $\psi: J \rightarrow X$ and $\sigma: J \rightarrow \mathbf{F}(X)$ be such that $\sigma(y) \stackrel{q_{\llcorner}}{\longrightarrow} \psi(y)$, for each $y \in J$. If $\mathcal{F} \in \mathbf{F}(J)$ and $\kappa \sigma \mathcal{F} \stackrel{q_{\mu}}{\rightarrow} x$, then $\psi \mathcal{F}^{q_{T(\mu, \nu)}} \stackrel{\text { ․ }}{\longrightarrow} x$.

For a fixed $T \in \mathbf{T}$, the full subcategory of PCS whose objects satisfy $\mathbf{F}_{T}$ is denoted by $\mathrm{F}_{T}$ PCS. The next three propositions summarize some already known results.

Proposition 2.2. [8]

(1) $F_{T} P C S$ is a bireflective subcategory of PCS, for every $T \in \mathbf{T}$;

(2) PTS $\subseteq F_{T} P C S \subseteq P P R S$, for every $T \in T$;

(3) If $T=\check{T}$, then $\mathrm{F}_{T} \mathrm{PCS}=\mathrm{PTS}$.

In [7], R. Lowen introduced the category AP of approach spaces which contains the categories TOP and MET (metric spaces and non-expansive maps) as full subcategories. In [6], R. Lowen and E. Lowen embedded AP in a quasitopos CAP of convergence approach spaces. As a consequence of 
the next proposition, both CAP and AP are bireflectively embedded in PCS.

Proposition 2.3[2]. Let $T$ be any strict $t$-norm.

(1) AP is isomorphic to the full subcategory of $\mathrm{F}_{T} P C S$ whose objects are left-continuous limit spaces.

(2) CAP is isomorphic to the full subcategory of PCS whose objects are left-continuous limit spaces.

In view of the first assertion of Proposition 2.3, we define objects in $\mathrm{F}_{T} \mathrm{PCS}$ to be $T$-approachable. In view of Proposition $2.2(3)$, the $\check{T}$-approachable probabilistic convergence spaces are precisely the probabilistic topological spaces, which yields a direct generalization of Proposition 1.2 (1).

Proposition 2.4[8]. For a probabilistic pretopological space $(X, \mathbf{q})$, and a $t$-norm $T$, the following statements are equivalent.

(1) $(X, q)$ is $T$-approachable.

(2) For arbitrary $\mu, \nu \in I$ and for each $V \in \mathcal{V}_{q_{T(\mu, \nu)}}(x)$, there exists $W \in \mathcal{V}_{q_{\mu}}(x)$ such that, for each $y \in W, V \in \mathcal{V}_{q_{\nu}}(y)$.

(3) For arbitrary $\mu, \nu \in I$ and $A \subseteq X, c l_{q_{\mu}}\left(c l_{g_{\nu}}(A)\right) \subseteq c l_{g_{T(\mu, \nu)}}(A)$.

\section{T-REGULARITY.}

A probabilistic convergence space $(X, \mathbf{q})$ is defined to be $T$-regular if it satisfies the axiom $\mathbf{R}_{T}$. The $T$-regular objects in PCS determine the full subcategory $\mathrm{R}_{T} \mathrm{PCS}$.

Theorem 3.1. Let $(X, \mathbf{q}) \in|P C S|, T \in \mathbf{T}$. Then $(X, \mathbf{q})$ is $T$-regular iff, for all $\mu, \nu \in I, \mathcal{F} \stackrel{\text { qu }}{\rightarrow} x$ implies $c l_{q_{\mu}} \mathcal{F} \stackrel{q_{T}(\mu, \nu)}{\longrightarrow} x$.

Proof. Assume that $(X, \mathbf{q})$ is $T$-regular, and let $\mathcal{F} \stackrel{q_{\nu}}{\rightarrow} x$. For arbitrary $\mu \in I$, let

$$
J=\left\{(\mathcal{G}, y): \mathcal{G} \in \mathbf{U}(X), y \in X, \mathcal{G} \stackrel{q_{\mu}}{\rightarrow} y\right\} .
$$

Define $\sigma: J \rightarrow \mathbf{F}(X)$ by $\sigma(\mathcal{G}, y)=\mathcal{G}$, and $\psi: J \rightarrow X$ by $\psi(\mathcal{G}, y)=y$. Then $\sigma(z) \stackrel{q_{\mu}}{\rightarrow} \psi(z)$ holds for all $z \in J$. For each $F \in \mathcal{F}$, let $S_{F}=\{(\mathcal{G}, y) \in J: F \in \mathcal{G}\}$, and let $S$ be the filter on $J$ with base $\left\{S_{F}: F \in \mathcal{F}\right\}$. One easily verifies that $\mathcal{F} \subseteq \kappa \sigma \mathcal{S}$, and hence $\kappa \sigma \mathcal{S} \stackrel{q_{\nu}}{\rightarrow} x$. By $\mathbf{R}_{T}$, it follows that $\psi(\mathcal{S})=c l_{q_{\mu}} \mathcal{F} \stackrel{q_{T(\mu, \nu)}}{\longrightarrow} x$, as desired.

Conversely, let $J, \sigma, \psi$, and $\mathcal{F} \in \mathbf{F}(J)$ be as in the statement of $\mathbf{R}_{T}$, and assume that $\kappa \sigma \mathcal{F} \stackrel{q_{\mu}}{\rightarrow} x$. Since $\sigma(y) \stackrel{q_{\nu}}{\rightarrow} \psi(y)$, for all $y \in J$, one may confirm that $c l_{q_{\nu}} \kappa \sigma \mathcal{F} \subseteq \psi \mathcal{F}$. But $c l_{q_{\nu}} \kappa \sigma \mathcal{F} \stackrel{q_{T}(\mu, \nu)}{\longrightarrow} x$ by hypothesis, and consequently $\psi \mathcal{F} \stackrel{q_{T}(\mu, \nu)}{\longrightarrow} x$. Thus $(X, \mathbf{q})$ satisfies $\mathbf{R}_{T}$. I

Corollary 3.2. Let $(X, \mathbf{q})$ be a probabilistic convergence space.

(1) If $T, T^{\prime} \in \mathbf{T}$ and $T^{\prime} \leq T$, then if $(X, \mathbf{q})$ is $T$-regular, it is also $T^{\prime}$-regular.

(2) If $(X, q)$ is $T$-regular for some $T \in \mathbf{T}$ and $\mathcal{F} \stackrel{q_{1}}{\rightarrow} x$, then $c l_{q_{\mu}} \mathcal{F} \stackrel{q_{\mu}}{\rightarrow} x$ holds for all $\mu \in I$.

In particular, if $(X, q)$ is $T$-regular, then $q_{1}$ is a regular convergence structure.

(3) $(X, \mathbf{q})$ is $\check{T}$-regular iff $(X, \mathbf{q})$ is componentwise regular (i.e., $q_{\mu}$ is a regular convergence structure for all $\mu \in I)$.

(4) $(X, q)$ is $\hat{T}$-regular iff both of the following hold:

(i) $\mathcal{F} \stackrel{q_{1}}{\rightarrow} x \Rightarrow c l_{q_{\mu}} \mathcal{F} \stackrel{q_{\mu}}{\rightarrow} x$, for all $\mu \in I$ 
(ii) $\mathcal{F} \stackrel{q_{\mu}}{\rightarrow} x \Rightarrow c l_{q_{1}} \mathcal{F} \stackrel{q_{\mu}}{\rightarrow} x$, for all $\mu \in I$

Proof. All of these results follow directly from Theorem 3.1. In particular, the first assertion in (2) follows by taking $\nu=1$, and the second by letting $\mu=\nu=1$. To prove (3), let $\mu=\nu$ and note that $\check{T}(\mu, \mu)=\mu \wedge \mu=\mu$.

In the subcategories PPRS and PTS of PCS, the characterization of $T$-regularity given in Theorem 3.1 can be reformulated as follows.

\section{Corollary 3.3.}

(1) $(X, \mathbf{q}) \in \mid$ PPRS $\mid$ is $T$-regular iff, for all $\mu, \nu \in I$ and $x \in X, \mathcal{V}_{q_{T(\mu, \nu)}}(x) \subseteq c l_{q_{\mu}}\left(\mathcal{V}_{q_{\nu}}(x)\right)$.

(2) $(X, \mathbf{q}) \in|\mathrm{PTS}|$ is $T$-regular iff the following holds for all $\mu, \nu \in I$ : If $x \in X$ and $A \subseteq X$ is a $q_{T(\mu, \nu)}$-closed set not containing $x$, then there is a $q_{\mu}$-open neighborhood $U$ of $x$ and a $q_{\nu}$-open neighborhood $V$ of $A$ such that $U \cap V=\emptyset$.

A probabilistic convergence space is said to be strongly regular if it is $\check{T}$-regular and weakly regular if it is $\hat{T}$-regular. It follows by Corollary $3.2(4)$ that every $(X, q) \in|\mathrm{PCS}|$ such that $q_{1}$ is discrete is weakly regular, demonstrating a wide gap between weak and strong regularity. Note that "strong regularity" according to our definition coincides with "regularity" as defined in [8].

Example 3.4. Let $T$ be the $t$-norm defined by $T(\mu, \nu)=\mu \nu$, let $X$ be any infinite set, and let $q$ be the probabilistic convergence structure defined by

$$
q_{\mu}= \begin{cases}\text { discrete topology, } & \mu \in\left(\frac{1}{2}, 1\right] \\ \text { cofinite topology, } & \mu \in\left(\frac{1}{4}, \frac{1}{2}\right] \\ \text { indiscrete topology, } & \mu \in\left[0, \frac{1}{4}\right]\end{cases}
$$

We obtain a $T$-regular space $(X, \mathbf{q})$ which is not strongly regular. However if we modify $\mathbf{q}$ only slightly to obtain $\mathbf{p}$ defined by

$$
p_{\mu}= \begin{cases}\text { discrete topology, } & \mu \in\left(\frac{1}{2}, 1\right] \\ \text { cofinite topology, } & \mu \in\left[\frac{1}{4}, \frac{1}{2}\right] \\ \text { indiscrete topology, } & \mu \in\left[0, \frac{1}{4}\right),\end{cases}
$$

the resulting probabilistic convergence space $(X, \mathbf{p})$ is weakly regular but not $T$-regular. I

Example 3.5. We borrow from [8] an example of a probabilistic convergence space which is strongly regular but not $T$-approachable for any $t$-norm $T$. Let $\lambda$ denote Lebesgue measure and $\tau$ the usual topology on $I=[0,1]$. Let $X$ be the set of all real-valued, Lebesgue-measurable functions on $I$, the convergence structure $q_{\mu}$ on $X$ is defined as follows: $\mathcal{F} \stackrel{q_{\mu}}{\rightarrow} f$ iff there is $A \subseteq I$ such that $\lambda(A) \leq 1-\mu$ and $\mathcal{F}(\nu) \stackrel{\tau}{\rightarrow} f(\nu)$, for all $\nu \in I-A$. One easily verifies that $\mathbf{q}=\left(q_{\mu}\right)$ is a probabilistic convergence structure on $X$.

For arbitrary $\mu \in I$, let $\mathcal{F} \stackrel{q_{\mu}}{\rightarrow} f$. Then there is $A \subseteq I$ such that $\lambda(A) \leq 1-\mu$ and $\mathcal{F}(\nu) \stackrel{\tau}{\rightarrow} f(\nu)$, for all $\nu \in I-A$. By regularity of $\tau, c l_{\tau} \mathcal{F}(\nu) \stackrel{\tau}{\rightarrow} f(\nu)$ for all $\nu \in I-A$, and $\left(c l_{q_{\mu}} \mathcal{F}\right)(\nu) \geq c l_{\tau}(\mathcal{F}(\nu))$ implies that $\left(c l_{q_{\mu}} \mathcal{F}\right)(\nu) \stackrel{\tau}{\rightarrow} f(\nu)$ for all $\nu \in I-A$. Thus $c l_{q_{\mu}} \mathcal{F} \stackrel{q_{\mu}}{\rightarrow} f$, establishing that $\mathbf{q}$ is strongly 
regular. The fact that $(X, \mathbf{q})$ is not $T$-approachable for any $T \in \mathbf{T}$ is proved in Example 3.13, [8].

Example 3.6. Let $X$ be the set described in Example 3.5 and let $Y$ be the set obtained from $X$ by identifying functions which are equal almost everywhere. Let $\mathbf{p}$ be the probabilistic convergence structure on $Y$ defined as follows: for each $\mu \in(0,1], \mathcal{F} \stackrel{p_{\mu}}{\rightarrow} f$ in $Y$ iff, for each $\alpha>0$ and $\epsilon<\mu$ there is $F \in \mathcal{F}$ such that, for each $g \in F, \lambda\{\nu \in I:|g(\nu)-f(\nu)|<\alpha\} \geq \epsilon$. For $\mu=1, p_{1}$ is convergence in probability. Let $T^{\prime}$ be the $t$-norm defined for $\mu, \nu \in I$ by $T^{\prime}(\mu, \nu)=\max \{\mu+\nu-1,0\}$. It is shown in Example 3.14, [8], that $(Y, \mathbf{p})$ is $T^{\prime}$-approachable. We shall now show that $(Y, \mathbf{p})$ is also $T^{\prime}$-regular.

Let $\alpha>0, \mu, \nu \in I$, and $\mathcal{F} \stackrel{p_{\mu}}{\rightarrow} f$. Let $T^{\prime}(\mu, \nu)=\eta$. If $\eta=0, c l_{\nu} \mathcal{F} \stackrel{p_{\eta}}{\rightarrow} f$ is clear. If $\eta>0$, let $\epsilon$ be a number such that $0<\epsilon<\eta$. Since $\eta=\mu+\nu-1$, we can choose $\epsilon_{1}<\mu$ and $\epsilon_{2}<\nu$ such that $\epsilon_{1}+\epsilon_{2}-1 \geq \epsilon$. Then $\mathcal{F} \stackrel{p_{\mu}}{\rightarrow} f$ implies there is $F \in \mathcal{F}$ such that $\lambda\left\{\xi \in I:|h(\xi)-f(\xi)|<\frac{\alpha}{2}\right\} \geq \epsilon_{1}$. If $g \in c l_{q_{\nu}} F$, there is $\mathcal{G} \stackrel{p_{\nu}}{\rightarrow} g$ with $F \in \mathcal{G}$; thus there is $G \in \mathcal{G}$ such that $\lambda\{\xi \in I:|k(\xi)-g(\xi)|<$ $\left.\frac{\alpha}{2}\right\} \geq \epsilon_{2}$ for all $k \in G$. Let $h^{\prime} \in G \cap F$. Then $\lambda\{\xi \in I:|g(\xi)-f(\xi)|<\alpha\} \geq \lambda\left\{\xi \in I:\left|g(\xi)-h^{\prime}(\xi)\right|+\right.$ $\left.\left|h^{\prime}(\xi)-f(\xi)\right|<\alpha\right\} \geq \lambda\left[\left\{\xi \in I:\left|g(\xi)-h^{\prime}(\xi)\right|<\frac{\alpha}{2}\right\} \cap\left\{\xi \in I:\left|h^{\prime}(\xi)-f(\xi)\right|<\frac{\alpha}{2}\right\}\right] \geq \epsilon_{1}+\epsilon_{2}-1 \geq \epsilon$. Therefore $c l_{p_{\nu}} \mathcal{F} \stackrel{p_{\eta}}{\rightarrow} f$, which establishes that $(Y, \mathrm{p})$ is $T^{\prime}$-regular.

In particular, $(Y, \mathrm{p})$ is weakly regular. However $(Y, \mathrm{p})$ fails to be $T$-regular for $T(\mu, \nu)=\mu \nu$. Indeed, let $\mathcal{F}=\dot{f}$, where $f=\chi_{I}$ is the characteristic function for $I$. Let $\mu=\nu=\frac{1}{2}$, let $\mathcal{G}=\dot{g}$, where $g=\chi_{\left[0, \frac{1}{2}\right]}$. Then $\mathcal{F} \stackrel{p_{\frac{1}{3}}^{\rightarrow}}{\rightarrow} \chi_{\left[\frac{1}{2}, 1\right]}$, and $\mathcal{G} \geq c l_{\frac{1}{2}} \mathcal{F}$. But $\mathcal{G}$ fails to $p_{\frac{1}{4}}$-converge to $\chi_{\left[\frac{1}{2}, 1\right]}$, and it follows that $(Y, \mathbf{p})$ is not $T$-regular.

Theorem 3.7. For a fixed $t$-norm $T$, let $\left\{\left(Y_{\alpha}, \mathrm{p}^{\alpha}\right): \alpha \in A\right\}$ be a collection of objects in $\mathrm{R}_{T} \mathrm{PCS}$. Let $X$ be a set and $f_{\alpha}: X \rightarrow Y_{\alpha}$ a function, for all $\alpha \in A$. If $q$ is the initial structure on $X$ relative to the families $\left\{\left(Y_{\alpha}, \mathbf{p}^{\alpha}\right): \alpha \in A\right\}$ and $\left\{f_{\alpha}: \alpha \in A\right\}$, then $(X, \mathbf{q})$ is $T$-regular.

Proof. As is noted in [8], for any $\nu \in I, \mathcal{F} \stackrel{q_{\nu}}{\rightarrow} x$ iff $f_{\alpha}(\mathcal{F}) \stackrel{p_{\alpha}^{\alpha}}{\rightarrow} f_{\alpha}(x)$, for all $\alpha \in A$. Thus for $\mu, \nu \in$ $I, \mathcal{F} \stackrel{q_{\nu}}{\rightarrow} x$ implies $c l_{p_{\nu}}\left(f_{\alpha}(\mathcal{F})\right) \rightarrow f_{\alpha}(x)$ in $\left(Y_{\alpha}, p_{T(\mu, \nu)}^{\alpha}\right)$, for all $\alpha \in A$. Since $f_{\alpha}:\left(X, q_{\mu}\right) \rightarrow\left(Y_{\alpha}, p_{\mu}^{\alpha}\right)$ is continuous for all $\mu \in I, c l_{p_{\mu}}\left(f_{\alpha}(\mathcal{F})\right) \subseteq f_{\alpha}\left(c l_{q_{\mu}}(\mathcal{F})\right)$, and hence $f_{\alpha}\left(c l_{q_{\mu}}(\mathcal{F})\right) \rightarrow f_{\alpha}(x)$ in $\left(Y_{\alpha}, p_{T(\mu, \nu)}^{\alpha}\right)$ holds for each $\alpha \in A$, since every $\left(Y_{\alpha}, \mathrm{p}^{\alpha}\right)$ is $T$-regular. Consequently, $c l_{q_{\mu}}(\mathcal{F}) \rightarrow x$ in $\left(X, q_{T(\mu, \nu)}\right)$, establishing that $(X, q)$ is $T$-regular.

Corollary 3.8. $T$-regularity is preserved under subspaces and arbitrary products in PCS. Furthermore, $\mathrm{R}_{T} \mathrm{PCS}$ is bireflectively embedded in PCS for any $T \in \mathbf{T}$.

Corollary 3.9. CONV and RCONV are bicoreflectively embedded in PCS and $R_{T}$ PCS, respectively, for any $T \in \mathbf{T}$.

Proof. In both cases the bicoreflector maps $(X, q)$ to $\left(X, q_{1}\right)$ and preserves the underlying function. Note that $q_{1}$ is regular whenever $q$ is $T$-regular by Corollary 3.2 (2). I

We have seen in Example 3.3 that $T$-regularity does not generally imply "componentwise regularity," and the question naturally arises whether there is some weaker property which every component structure of a $T$-regular probabilistic convergence structure must satisfy. Indeed, there is such a property which we define as follows: a convergence space $(X, q)$ is symmetric if, for all $x, y \in X, \dot{y} \stackrel{q}{\rightarrow} x$ implies $\dot{x} \stackrel{q}{\rightarrow} y$. Note that every regular convergence structure is symmetric. 
Proposition 3.10. A probabilistic convergence space $(X, \mathbf{q})$ which is $T$-regular for any $T \in \mathbf{T}$ has the property that each component convergence space $\left(X, q_{\mu}\right)$ is symmetric.

Proof. Let $\mu \in I$ and let $\dot{y} \stackrel{q_{\mu}}{\rightarrow} x$. Let $J=X$, let $\psi$ be the identity map on $X$, and let $\sigma: X \rightarrow \mathbf{F}(X)$ be defined by $\sigma(x)=\dot{y}$ and $\sigma(z)=\dot{z}$, for $z \neq x$. Then $\kappa \sigma \dot{x}=\dot{y}$, and by $\mathbf{R}_{T}, \dot{x} \stackrel{q_{\mu}}{\rightarrow} y$, since $\mu=T(\mu, 1)$. I

We close this section with a simple characterization of those probabilistic convergence spaces which are simultaneously $T$-approachable and $T$-regular.

Proposition 3.11. $(X, \mathbf{q}) \in\left|\mathrm{F}_{T} \mathrm{PCS}\right| \cap\left|\mathrm{R}_{T} \mathrm{PCS}\right|$ iff the following conditions are satisfied:

(1) $c l_{q_{\mu}}\left(\mathcal{V}_{q_{\nu}}(x)\right) \rightarrow x$ in $\left(X, q_{T(\mu, \nu)}\right)$, for all $\mu, \nu \in I$ and $x \in X$.

(2) $c_{q_{\mu}}\left(c l_{q_{\nu}}(A)\right) \subseteq c l_{q_{T(\mu, \nu)}}(A)$, for all $\mu, \nu \in I$ and $A \subseteq X$.

The proof is an easy consequence of Proposition 2.4 and Theorem 3.1.

\section{ALTERNATE FORMULATIONS OF THE AXIOMS.}

The results of the preceding section pertaining to $\mathbf{R}_{T}$, combined with those of [2] and [8] involving $\mathbf{F}_{T}$, demonstrate the usefulness of these axioms in the study of probabilistic convergence spaces. Furthermore $\mathbf{R}_{T}$, when translated under the isomorphism mentioned in Proposition 2.3, has important ramifications in the study of approach spaces, convergence approach spaces, and related categories which we shall discuss elsewhere.

In working with axioms or definitions based on filters, it is useful to know when "filter" can be replaced by "ultrafilter" with no resulting loss of generality. This is true, for instance, in defining "Hausdorf" in the setting of convergence spaces; an additional illustration is found in Proposition 1.3. In this concluding section we show that Proposition 1.3 can be generalized to the axioms $\mathbf{F}_{T}$ and $\mathbf{R}_{T}$ for arbitrary probabilistic convergence spaces. Furthermore, by restricting $\mathbf{F}_{T}$ and $\mathbf{R}_{T}$ to the category PPSS of probabilistic pseudotopological spaces, these axioms can be given equivalent formulations based entirely on ultrafilter convergence.

For a probabilistic convergence space $(X, \mathrm{q})$ and a $t$-norm $T$, let $\mathrm{F}_{T}^{*}$ be, the axiom obtained when "F $(X)^{n}$ is replaced by " $\mathbf{U}(X)$ " in the axiom $\mathbf{F}_{T}$. Furthermore, let $\mathbf{F}_{T}^{* *}$ be the axiom obtained when "F $(J)$ " is replaced by " $\mathbf{U}(J)$ " in the axiom $\mathbf{F}_{T}^{*}$. In exactly the same way, we derive $\mathbf{R}_{T}^{*}$ from $\mathbf{R}_{T}$ and $\mathbf{R}_{T}^{* *}$ from $\mathbf{R}_{T}^{*}$. It is obvious that $\mathbf{F}_{T} \Rightarrow \mathbf{F}_{T}^{*} \Rightarrow \mathbf{F}_{T}^{* *}$ and $\mathbf{R}_{T} \Rightarrow \mathbf{R}_{T}^{*} \Rightarrow \mathbf{R}_{T}^{* *}$.

Theorem 4.1. For $(X, \mathbf{q}) \in|\mathrm{PCS}|$ and $T \in \mathbf{T}, \mathbf{F}_{T} \Longleftrightarrow \mathbf{F}_{T}^{*}$ and $\mathbf{R}_{T} \Longleftrightarrow \mathbf{R}_{T}^{*}$.

Proof. Assume $\mathbf{F}_{T}^{*}$. By Proposition 2.4, it suffices to show that $(X, \mathbf{q})$ is a probabilistic pretopological space which satisfies: $c l_{q_{\mu}}\left(c l_{q_{\nu}}(A)\right) \subseteq c l_{q_{T(\mu, \nu)}}(A)$, for all $\mu, \nu \in I$ and $A \subseteq X$. The proof of Proposition 3.5, [8], estabishes that if $(X, \mathbf{q})$ satisfies $\mathbf{F}_{T}^{*}$, then $(X, \mathbf{q})$ is pretopological. To prove the assertion about closures, let $x \in \operatorname{cl}_{q_{\mu}}\left(c l_{q_{\nu}}(A)\right)$; then there is an ultrafilter $\mathcal{H} \stackrel{q_{\mu}}{\rightarrow} x$ such that $c l_{q_{\nu}}(A) \in \mathcal{H}$. Let $J=X$ and $\psi(y)=y$, for all $y \in X$. If $y \in c l_{q_{\nu}}(A)$, there is an ultrafilter $\mathcal{G}_{y} \stackrel{q_{\nu}}{\rightarrow} y$ such that $A \in \mathcal{G}_{y}$. Define $\sigma(y)=\mathcal{G}_{y}$, for $y \in c l_{q_{\nu}}(A)$ and $\sigma(y)=\dot{y}$, if $y \notin c l_{q_{\nu}}(A)$. If follows that $A \in \kappa \sigma \mathcal{H}$. By $\mathrm{F}_{T}^{*}, \kappa \sigma \mathcal{H}^{q_{T(\mu, \nu)}} x$, and therefore $x \in \operatorname{cl}_{q_{T(\mu, \nu)}}(A)$. 
Next, assume $\mathbf{R}_{T}^{*}$. To establish $\mathbf{R}_{T}$, it suffices to prove the characterization of $\mathbf{R}_{T}$ given in Theorem 3.1. But in the first half of the proof of Theorem 3.1, we observe that $\sigma(z) \in \mathrm{U}(X)$ for every $z \in J$. Thus this same proof remains valid when $\mathbf{R}_{T}$ is replaced by $\mathbf{R}_{T}^{*}$. I

Lemma 4.2. Let $J, \sigma$, and $\mathcal{F} \in \mathbf{F}(J)$ be as in the statement of $\mathbf{F}_{T}^{*}$. If $\mathcal{U} \in \mathbf{U}(X)$ and $\mathcal{U} \geq \kappa \sigma \mathcal{F}$, then there exists a $\mathcal{G} \in \mathrm{U}(J), \mathcal{G} \geq \mathcal{F}$ such that $\kappa \sigma \mathcal{G}=\mathcal{U}$.

Proof. Let $\mathcal{U} \in \mathrm{U}(X)$ be such that $\mathcal{U} \geq \kappa \sigma \mathcal{F}$. For $A \in \mathcal{U}$, define $H_{A}=\{y \in J: A \in \sigma(y)\}$. Observe that $H_{A} \cap F \neq \emptyset$ for all $F \in \mathcal{F}$ and all $A \in \mathcal{U}$; otherwise, for some $F \in \mathcal{F}, X \backslash A \in \sigma(y)$ for all $y \in F$. But this implies $X \backslash A \in \kappa \sigma \mathcal{F}$, which is a contradiction. Let $\mathcal{H}$ be the filter generated by $\left\{H_{A}: A \in \mathcal{U}\right\}$, and let $\mathcal{G}$ be any ultrafilter on $J$ with the property that $\mathcal{G} \geq \mathcal{F} \vee \mathcal{H}$. It is easy to verify that $\kappa \sigma \mathcal{G}=\mathcal{U}$.

Theorem 4.3. For $(X, \mathbf{q}) \in|P P S S|$ and $T \in \mathbf{T}, \mathbf{F}_{T} \Longleftrightarrow \mathbf{F}_{T}^{* *}$.

Proof. Assume $\mathbf{F}_{T}^{* *}$. Let $J, \sigma, \psi$, and $\mathcal{F} \in \mathbf{U}(J)$ be as in the statement of $\mathbf{F}_{T}^{*}$, and assume that $\psi \mathcal{F} \stackrel{q_{\mu}}{\rightarrow} x$. Let $\mathcal{U} \in \mathrm{U}(X)$ be such that $\mathcal{U} \geq \kappa \sigma \mathcal{F}$. By Lemma 4.2 , there exists a $\mathcal{G} \in \mathbf{U}(J)$, such that $\mathcal{G} \geq \mathcal{F}$ and $\mathcal{U}=\kappa \sigma \mathcal{G}$. Hence, $\psi \mathcal{G} \stackrel{q_{\mu}}{\rightarrow} x$, and so $\mathcal{U}=\kappa \sigma \mathcal{G} \stackrel{q^{\boldsymbol{q}_{(\mu, \nu)}}}{\rightarrow} x$, by $\mathbf{F}_{\tilde{T}}^{* *}$. Since $\boldsymbol{q}_{\boldsymbol{\tau}(\mu, \nu)}$ is pseudotopological by assumption, it follows that $\kappa \sigma \mathcal{F}^{q_{T(\mu, \nu)}} \stackrel{\text { Thus, }}{\longrightarrow}(X, \mathbf{q})$ satisfies $\mathbf{F}_{T}^{*}$. Theorem 4.1 now implies that $(X, \mathbf{q})$ satisfies $\mathbf{F}_{T} . \mathbf{I}$

Lemma 4.4 Let $J, \sigma, \psi$, and $\mathcal{F} \in \mathbf{F}(J)$ be as in the statement of $\mathbf{R}_{T}$. If $\mathcal{U} \in \mathbf{U}(X)$ and $\mathcal{U} \geq \psi(\mathcal{F})$, then there exists a $\mathcal{G} \in \mathbf{U}(J)$ such that $\kappa \sigma \mathcal{G} \geq \kappa \sigma \mathcal{F}$ and $\mathcal{U}=\psi(\mathcal{G})$.

Proof. Let $\mathcal{U} \in \mathbf{U}(X)$ be such that $\mathcal{U} \geq \psi(\mathcal{F})$. For $A \in \mathcal{U}$ and $F \in \mathcal{F}$, define

$$
G_{\boldsymbol{A}, \boldsymbol{F}}=\{y \in F: \psi(y) \in A \cap \psi(F)\},
$$

and let $\mathcal{G}$ be any ultrafilter on $J$, with the property that $\mathcal{G}$ is finer than the filter generated by $\left\{G_{A, F}: A \in \mathcal{U}\right.$ and $\left.F \in \mathcal{F}\right\}$. Since $\mathcal{G} \geq \mathcal{F}, \kappa \sigma \mathcal{G} \geq \kappa \sigma \mathcal{F}$. Also, $\psi\left(G_{A, F}\right) \subseteq A$, for all $F \in \mathcal{F}$ and all $A \in \mathcal{U} ;$ thus $\psi(\mathcal{G})=\mathcal{U}$.

Theorem 4.5. For $(X, \mathbf{q}) \in \mid$ PPSS $\mid$ and $T \in \mathbf{T}, \mathbf{R}_{T}^{* *} \Longleftrightarrow \mathbf{R}_{T}$.

Proof. Assume $\mathbf{R}_{\vec{T}}^{* *}$. Let $J, \sigma, \psi$, and $\mathcal{F} \in \mathbf{F}(J)$ be as in the statement of $\mathbf{R}_{T}^{*}$, and assume that $\kappa \sigma \mathcal{F} \stackrel{q_{\mu}}{\rightarrow} x$ for some $x \in X$. Let $\mathcal{U} \in \mathrm{U}(X)$ be such that $\mathcal{U} \geq \psi(\mathcal{F})$. By Lemma 4.4, there exists a $\mathcal{G} \in \mathrm{U}(J)$ such that $\kappa \sigma \mathcal{G} \geq \kappa \sigma \mathcal{F}$ and $\psi(\mathcal{G})=\mathcal{U}$. Hence, $\kappa \sigma \mathcal{G} \stackrel{q_{\mathcal{H}}}{\longrightarrow} x$, and so $\mathcal{U}=\psi(\mathcal{G}) \stackrel{{ }^{q_{(\mu, \nu)}}}{\longrightarrow} x$, by $\mathbf{R}_{T}^{* *}$. Since $q_{T(\mu, \nu)}$ is pseudotopological by assumption, it follows that $\psi(\mathcal{F}) \stackrel{q^{T_{(\mu, \nu)}}}{\rightarrow} x$. Thus, $(X, \mathbf{q})$ satisfies $\mathbf{R}_{T}^{*}$. Theorem 4.1 now implies that $(X, \mathbf{q})$ satisfies $\mathbf{R}_{T}$. I

Corollary 4.6. Let $(X, \mathbf{q}) \in|\mathrm{PPSS}|, T \in \mathbf{T}$. Then $(X, \mathbf{q})$ is $T$-regular iff, whenever $\mu, \nu \in I, \mathcal{F} \in$ $\mathrm{U}(X)$, and $\mathcal{F} \stackrel{q_{\nu}}{\rightarrow} x$, then $c l_{q_{\mu}} \mathcal{F}^{q_{T}(\mu, \nu)} \rightarrow$

Proof. Use the second half of the proof of Theorem 3.1 to establish $\mathbf{R}_{T}^{* *}$. 


\section{REFERENCES}

[1 ] H.J. Biesterfeldt, Jr., "Regular Convergence Spaces," Indag. Math. 28 (1966), 605-607.

[2 ] P. Brock and D.C. Kent, "Approach Spaces, Limit Tower Spaces, and Probabilistic Convergence spaces," Applied Categoried Structures (To appear).

[3 ] C.H. Cook and H.R. Fischer, "Regular Convergence Structure," Math. Ann. 174 (1967), 1-7.

[4 ] L.C. Florescu, "Probabilistic Convergence Structures," Aequations Math. 38 (1989), 123-145.

[5 ] D.C. Kent and G.D. Richardson, "Convergence Spaces and Diagonal Conditions," Top. and its Appl. (To appear).

[6 ] E. Lowen and R. Lowen, "A Quasitopos Containing CONV and MET as Full Subcategories," Intl. J. Math. and Math. Sci. II (1988), 417-438.

[7 ] R. Lowen, "Approach Spaces: A Common Supercategory of TOP and MET," Math. Nachr. 141 (1989), 183-226.

[8 ] G.D. Richardson and D.C. Kent, "Probabilistic Convergence Spaces," J. Austral. Math. Soc. (To appear).

[9 ] B. Schweizer and A. Sklar, Probabilistic Metric Spaces, North Holland Publ. Co., New York, 1983. 


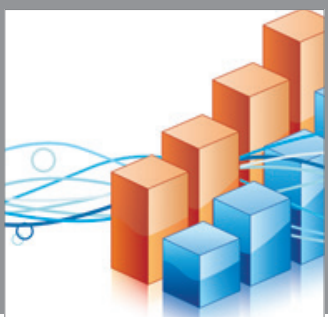

Advances in

Operations Research

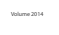

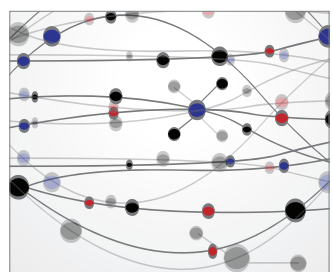

\section{The Scientific} World Journal
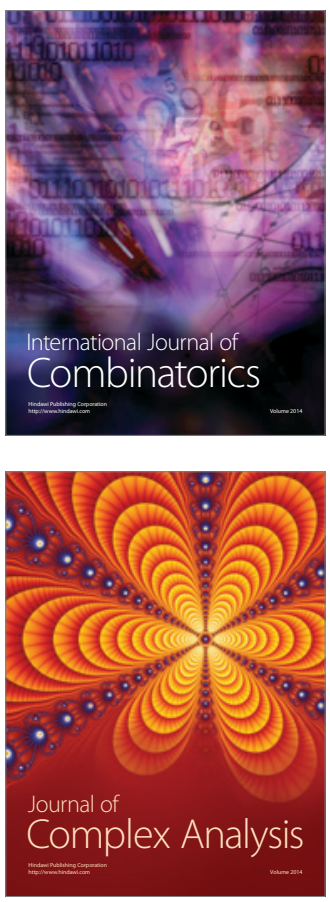

International Journal of

Mathematics and

Mathematical

Sciences
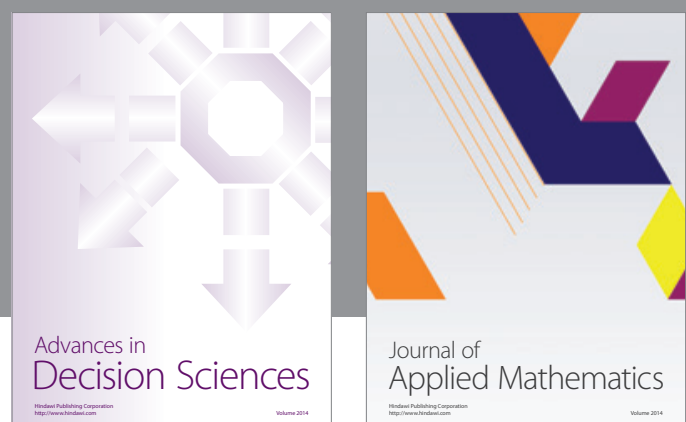

Journal of

Applied Mathematics
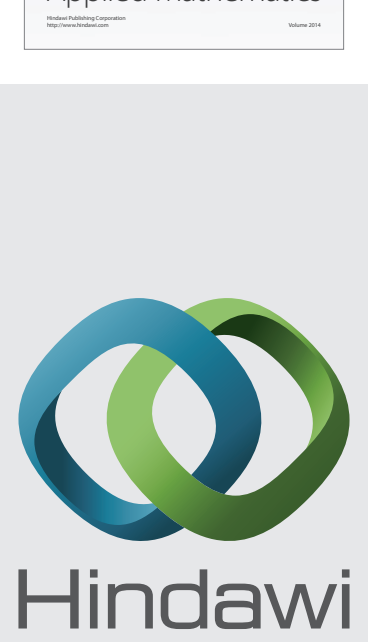

Submit your manuscripts at http://www.hindawi.com
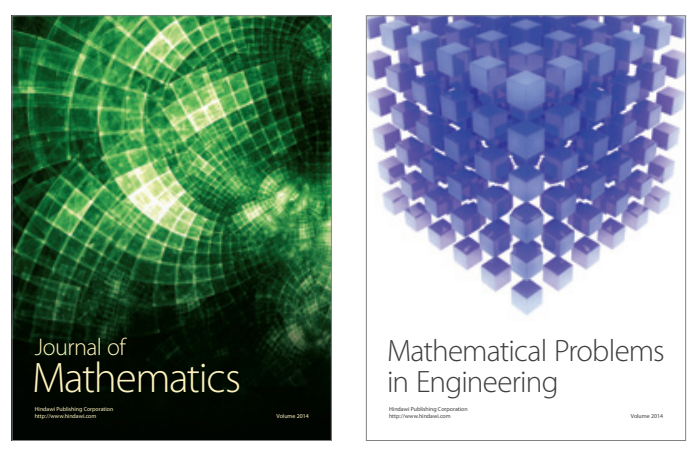

Mathematical Problems in Engineering
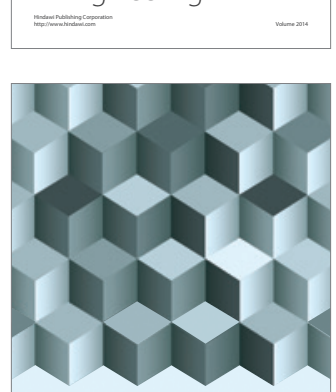

Journal of

Function Spaces
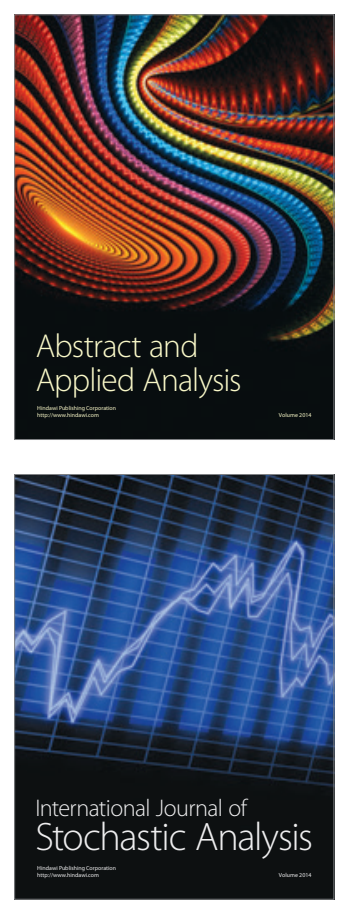

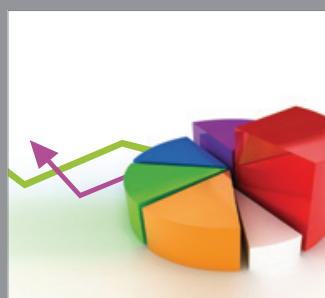

ournal of

Probability and Statistics

Promensencen
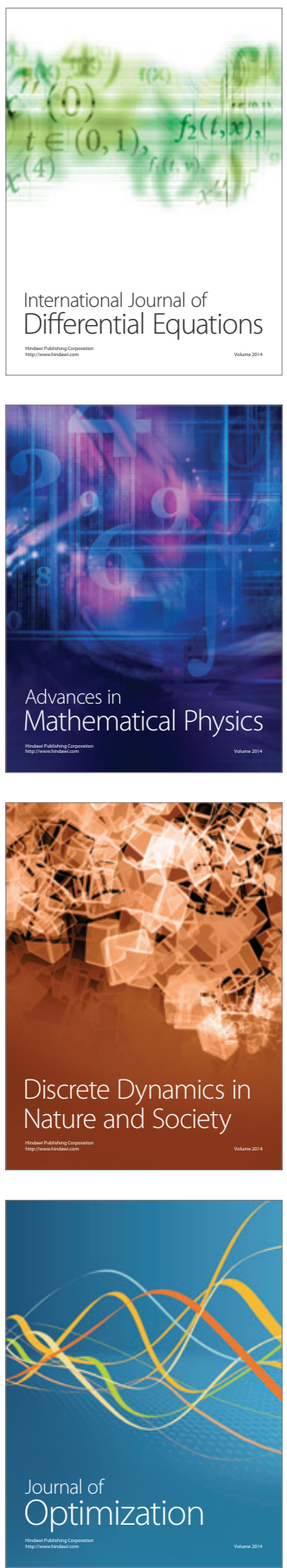